\title{
Ionospheric Variations in the Period Range of Days to Tens of Days Deduced from HF Doppler Observation
}

\author{
Takashi Shibata $^{1}$, Yasuo Yoshimura ${ }^{1}$, Toshio Ogawa ${ }^{2}$, and Takashi OkuZawa ${ }^{1}$ \\ ${ }^{1}$ Department of Electronic Engineering, Denki-Tsushin University, Chofu-shi, Tokyo 182, Japan \\ ${ }^{2}$ Department of Physics, Kochi University, Kochi 780, Japan
}

(Received October 14, 1988; Revised April 20, 1989)

\section{Introduction}

As is well known, HF Doppler (HFD) frequency variations of the ionosphere correspond to ionospheric phase path changes, which should be ascribed to traveling ionospheric disturbances, solar flares (UV and X-ray), magnetic pulsations, and geomagnetic sudden commencements, as well as sudden impulses, etc. (DAVIES and BAKER, 1966; OKUZAWA and DAVIES, 1981; KIKUCHI et al., 1985). Therefore, it is possible that the HFD variation may be a manifestation of solarterrestrial activity in any sense.

This paper presents the result of spectrum analyses of the ionospheric variations in the period range of a few days to tens of days observed by the HFD method for nearly the entire year of 1986. For comparison purposes, the spectrum analyses have been done on the foF 2 data and the horizontal component $(H)$ data of the geomagnetic field, which were obtained during the same observation period as well.

It has been established that there are characteristic variations in that period range, if spectrum analyses are made on the data of total electron content, critical frequencies and virtual heights of the ionosphere, etc. (e.g., NOONKESTER, 1965; TITHERIDGE, 1971). According to them, the predominant spectral components are: the solar rotation period of around 27 days, the semi-synodical period of around 15 days, and several other periods shorter than 20 days or so related to geomagnetic storm activity. The immediate objective of the present study is therefore to examine whether or not these spectral components can also be detected from the HFD records.

Spectrum analyses of the HFD variations have so far been restricted to the period range from several minutes to a few hours, because we used to focus our attention on the ionospheric response to atmospheric acoustic-gravity waves (e.g., Shibata and OKUZAWA, 1983; WALDOCK and JONES, 1986). Hence, the present study, as far as we know, is the first concerning the spectral features of HFD variations in the long-period range of days to tens of days. 


\section{Observation and Data}

The HFD records have been provided by our continuous reception at Kochi $\left(33.5^{\circ} \mathrm{N}, 133.4^{\circ} \mathrm{E}\right)$ of the $\mathrm{JJY} 8 \mathrm{MHz}$ standard wave transmitted from Nazaki $\left(36.2^{\circ} \mathrm{N}, 139.9^{\circ} \mathrm{E}\right)$, from February to December of 1986 . The surface distance of the propagation path is about $659 \mathrm{~km}$, and the height of the ionospheric reflection point for the $8 \mathrm{MHz}$ waves can be estimated to range from 100 to $140 \mathrm{~km}$ in the daytime. The original HFD records are in digital form with a sampling time interval of $10 \mathrm{sec}$. We have divided them into consecutive subsets of 10 min duration, and have calculated the median value for each. Hourly values have been defined in terms of the average of such median values of six subsets equally distributed around each integral hour. Our analyses are limited here to the noon hours, because good quality data are available for the daytime only. Thus, we will treat three cases referring to the local time $\left(135^{\circ}\right.$ EST: called hereafter JST) of $11 \mathrm{~h}, 12 \mathrm{~h}$, and $13 \mathrm{~h}$ every day.

Intending to compare them with the HFD results, we have also done spectrum analyses on the hourly value data of $f o F 2$ and $H$ from Kokubunji $\left(35.7^{\circ} \mathrm{N}, 139.5^{\circ} \mathrm{E}\right)$ and Kakioka $\left(36.2^{\circ} \mathrm{N}, 140.2^{\circ} \mathrm{E}\right)$, respectively, for the same data period as the HFD. We have no missing data sections of the $H$ values, whereas there were missing sections of about $16 \%$ of the foF 2 and about $12 \%$ of the HFD values for the whole data intervals ( 334 days) of the present analyses; The missing data intervals have been interpolated to be reasonably filled by the AKIMA method (1970). The standard deviations of the complete data set of HFD, foF 2 and $H$ thus arranged amount to $4.9 \times 10^{-2} \mathrm{~Hz}, 1.4 \mathrm{MHz}$ and $23.6 \mathrm{nT}$, respectively.

Although $f_{O} E$ data would be more adequate to be compared with the HFD in the present case, we dared to use the foF 2 as a representative of the ionospheric parameters, because the foE from Kokubunji in the same observation period contained more missing data sections than the latter. This will be justified to some extent by the following: (1) for ionospheric variations having time scales of the order of one to ten days, there will scarcely be a difference between the foF 2 and the $f o E$ as far as their effective variability is concerned, and (2) the surface distance between the mid-path point of the HFD wave and Kokubunji amounts to about $280 \mathrm{~km}$, so that direct comparison between the $f o E$ from Kokubunji and the HFD at Kochi will not necessarily be significant. It goes without saying that the $H$ record should directly reflect the geomagnetic activity.

\section{Spectrum Analyses and Results}

The maximum entropy method (MEM) is employed in this study. The sampling time of the input data is first fixed as one day, and then the length of the data window is chosen to be 180 days (i.e., 60 days $\times 3$ ), so that we have a reasonable power estimate of the wave period up to 60 days. By successively shifting the window 10 days throughout the whole data set, we have 16 sets of sample data for each of the above three different kinds of data. From our experience of spectrum 

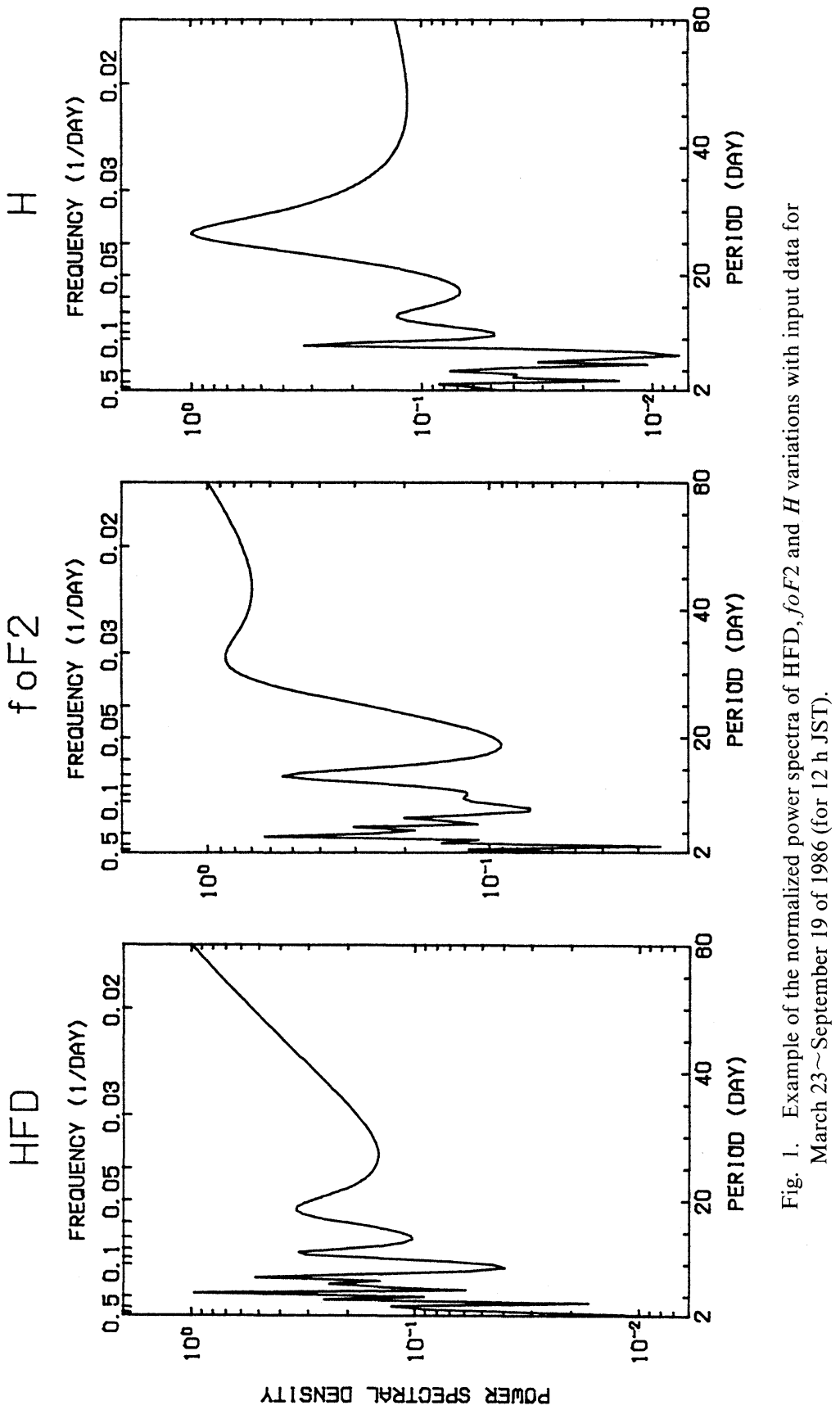
anaiyses on HFD data, the prediction error filter number for the MEM is chosen to be $20 \%$ of the window length.

Figure 1 shows an example of the resultant power spectra of the HFD, foF 2 and $H$ for March 23 to September 19, 1986 (for $12 \mathrm{~h} \mathrm{JST).} \mathrm{It} \mathrm{is} \mathrm{evident} \mathrm{from} \mathrm{the}$ figure that there are several dominant spectral peaks, with common wave periods among either two or three kinds of the data.

In order to elucidate the spectral peak distribution, the percent occurrence of the peaks against the wave period has been calculated, where the percent occurrence means the appearance rate of spectral peaks for the whole of the obtained spectra $(16 \times 3$ (i.e., for three JST's) $=48$ for each of the three kinds of data) with allowance of \pm 0.5 day period. The result for the HFD observation is shown in Fig. 2 by histogram. We can readily observe from this figure that the percent occurrence shows predominance in the period range of 3 to 13 days and around 16 21 days. We note also that few occurrences can be observed at periods longer than 22 days. Figure 3 is the same as Fig. 2 but for both $f_{o F}$ and $H$. It is seen that unlike the HFD case, the dominant spectral peaks for the latter two cases are roughly separated into three groups; i.e., the first group has periods below about 11 days, the second around $13 \sim 16$ days, and the last around $23 \sim 28$ days. It is highly probable that the latter two groups essentially originate from the semi-synodic variation $(\sim 15$ days) and the solar rotation $(\sim 27$ days $)$, respectively. The percent occurrence for the fo $F 2$ spectra around the 27 day period is observed to be relatively smaller than that of the $H$. This may be due to the low activity of the sun for 1986, a year of minimum sunspot number; this is consistent with NOONKESTER's (1965) statement that for foF 2 a significant spectral peak at a 27 day period was hardly observed during sunspot minimum. Comparison between the features of Figs. 2 and 3 makes us recognize that in the period range of about $13 \sim 30$ days, the occurrence distribution

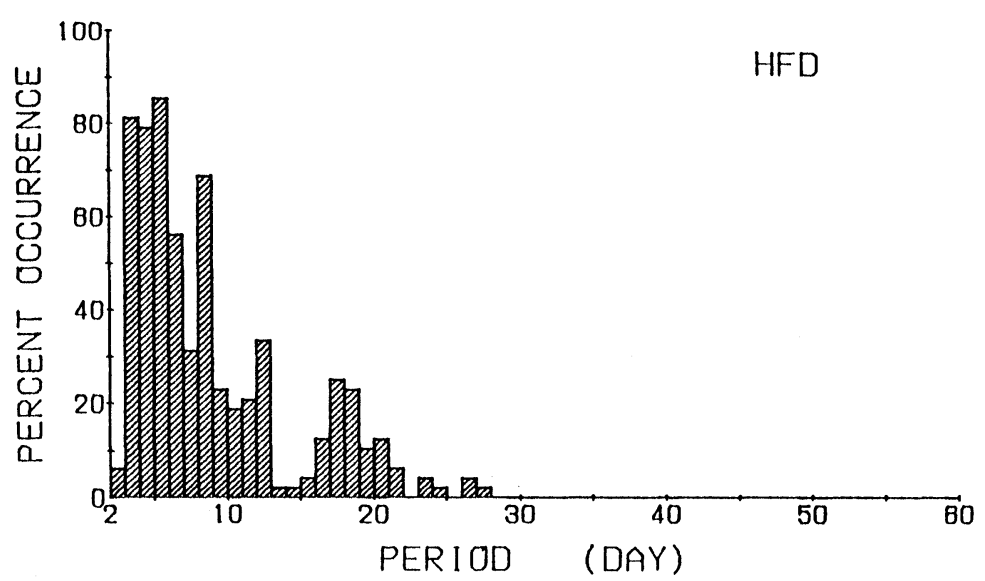

Fig. 2. Occurrence histogram of spectral peaks vs the wave period for HFD for February $\sim$ December of 1986. 


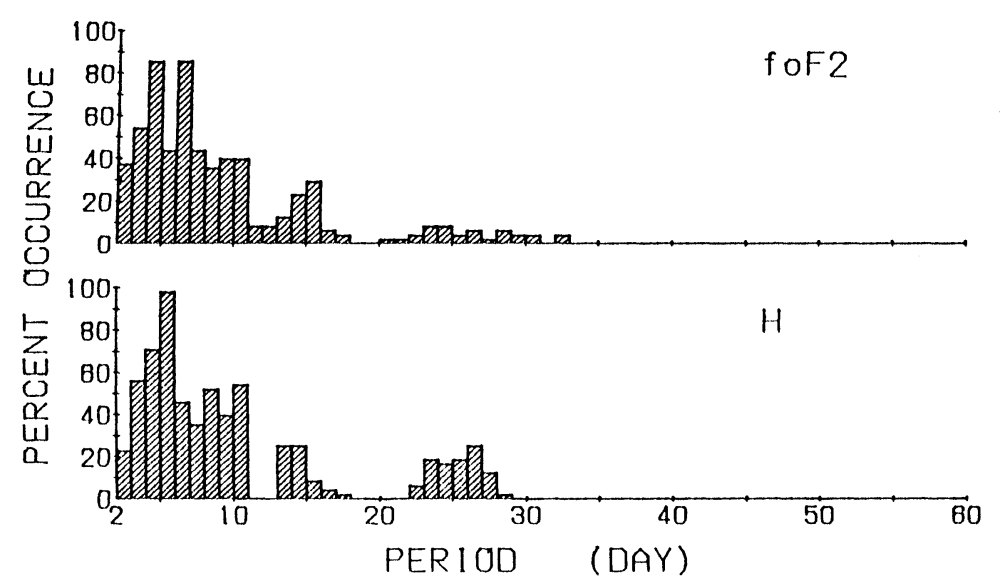

Fig. 3. The same as Fig. 2 but for the foF 2 and the $H$ data.

of spectral peaks in the HFD case is quite different from those of both the foF 2 and the $H$, in the sense that the former is characterized by its predominance around the 16 21 day period, whereas the latter two are not.

In the periods shorter than about 11 days, high-level features of the percent occurrence are common among the three sets of data, although the individual positions of the occurrence peaks are not necessarily the same among the three. This leads us to the inference that the HFD spectral peaks in this period range may be consistent with those of the $f o F 2$ and/or the $H$. We have therefore performed cross-spectral analyses on pair data of both HFD-foF 2 and HFD- $H$ using multichannel MEM (SHIBATA, 1987), and then calculated the percent rate of coherence peaks in the HFD spectra with either the $f o F 2$ or the $H$; we have calculated only for the wave periods in which the percent occurrence of the HFD spectral peaks in Fig. 2 exceeds $10 \%$, and have taken up the cases in which the coherence takes values greater than 0.8 in the period range of 2 to 35 days, outside which no spectral peaks have been observed. The results are shown in Fig. 4. We can see from this figure that the percent rate of coherence peaks is significant in the period range of $3 \sim 12$ days for the HFD-foF 2 pair and also $3 \sim 9$ days for the HFD- $H$ pair. This will endorse to a considerable extent our preceding expectation.

\section{Concluding Remarks}

The most important factor in dealing with HFD records is the frequency stability of the observation system; the stability is expected to depend mainly on that of the reference oscillator used in the receiver. Our crystal oscillator has a stability as good as $5 \times 10^{-9} /$ day at the primary stage of $5 \mathrm{MHz}$. The probable range of the frequency variation in our observation system, therefore, is smaller than the standard deviation of the HFD data we used. 


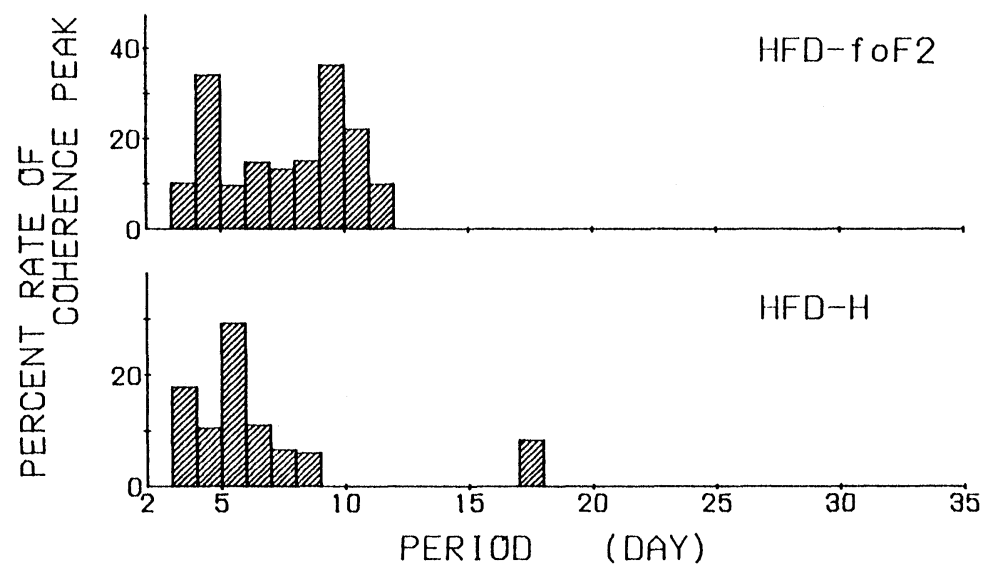

Fig. 4. Occurrence rate of the coherence peaks for the HFD spectra with the foF2 (upper) and the $H$ (lower).

The spectrum analyses have shown that our HFD observation surely detected ionospheric variations in the period range of both $3 \sim 13$ and $16 \sim 21$ days. Unfortunately, a 27 day period has not been observed in the HFD as well as the foF2 spectra; this will probably be ascribed to minimum solar activity. The variations with the periods of $3 \sim 13$ days have been found to be correlated to some extent with those of the $f_{o} F 2$ and/or the $H$; a kind of geomagnetic origin suggested by TITHERIDGE (1971) might be responsible for these ionospheric variations. Dominant spectra in the period range of 16 21 days are supposed to be peculiar to the HFD. Their real nature will not be revealed unless further spectral analyses are made on the HFD records for other observation periods.

The present analyses have dealt with the HFD signals of the E-region ionosphere. Therefore, the HFD signals of the $F$ region will be more adequate to be compared with the $f o F 2$. This is now being undertaken by us using the HFD data at Chofu station, and the result will be reported in the near future.

The $f_{O} F 2$ and the $H$ data used in this study were provided by the Communications Research Laboratory, Ministry of Posts and Telecommunications of Japan, and Kakioka Magnetic Observatory, Japan Meteorological Agency, respectively.

\section{REFERENCES}

Aкima, H., A new method of interpolation and smooth curve fitting based on local procedures, J. Assoc. Comput. Machin., 17, 589-602, 1970.

DAVIES, K. and D. M. BAKER, On frequency variations of ionospherically propagated HF radio signals, Radio Sci., 1, 545-556, 1966.

KIKUCHI, T., T. ISHIMINE, and H. SUGIUCHI, Local time distribution of HF Doppler frequency deviations associated with storm sudden commencements, J. Geophys. Res., 90, 4389-4393, 1985. 
NoOnkester, V. R., $E$ - and $F$-region variations near periods of 27 and 15 days at Washington D. C., $J$. Atmos. Terr. Phys., 27, 605-616, 1965.

Okuzawa, T. and K. Davies, Pulsations in total columnar electron content, J. Geophys. Res., 86, 1355-1363, 1981.

Shibata, T., Application of multichannel maximum entropy spectral analysis to the HF Doppler data of medium-scale TID, J. Geomag. Geoelectr., 39, 247-260, 1987.

Shibata, T. and T. Okuzawa, Horizontal velocity dispersion of medium-scale travelling ionospheric disturbances in the F-region, J. Atmos. Terr. Phys., 45, 149-159, 1983.

Titheridge, J. E., The spectrum of electron content fluctuations in the ionosphere, Planet. Space Sci., 19, 1593-1608, 1971.

Waldock, J. A. and T. B. Jones, HF Doppler observations of medium-scale travelling ionospheric disturbances at mid-latitudes, J. Atmos. Terr. Phys., 48, 245-260, 1986. 